\title{
Comparing consistency of clear corneal incisions using a traditional diamond keratome and a newly designed diamond keratome
}

This article was published in the following Dove Press journal:

Clinical Ophthalmology

31 July 2015

Number of times this article has been viewed

\section{Russell J Swan \\ Julia M Byrd \\ Daniel I Bettis \\ Randall J Olson}

Department of Ophthalmology and Visual Sciences, John A. Moran Eye Center, University of Utah, Salt Lake City, UT, USA
Correspondence: Randall J Olson John Moran Eye Center, 65 Mario Capecchi Drive, Salt Lake City, UT 84I32, USA

Tel +I 80I 5856622

Fax + 1 80I 58I3357

Email randallj.olson@hsc.utah.edu
Purpose: To compare the consistency of incision architecture utilizing a traditional diamond keratome and a newly designed diamond keratome.

Methods: We used a traditional diamond keratome and newly designed diamond keratome to create clear corneal incisions in human cadaveric donor eyes. Three surgeons with varying levels of experience made 30 incisions with each keratome; and the wound architecture was measured including incision lengths, epithelial and endothelial widths, and the central epithelial incision's deviation from a straight line entrance.

Results: The mean absolute difference in right and left incision lengths (traditional: $0.182 \pm$ $0.158 \mathrm{~mm}$ and new $0.088 \pm 0.077 \mathrm{~mm}[P<0.003])$, mean absolute difference in epithelial and endothelial incision width (traditional: $0.181 \pm 0.144 \mathrm{~mm}$ and new $0.080 \pm 0.092 \mathrm{~mm}[P<0.002]$ ), endothelial incision central deviation from a straight line (traditional: $0.128 \pm 0.242 \mathrm{~mm}$ and new $-0.046 \pm 0.124 \mathrm{~mm}[P<0.001])$ were all significantly more consistent with the newly designed diamond keratome than with the traditional diamond keratome.

Conclusion: The newly designed diamond keratome creates a more consistent clear corneal incision than a traditional diamond keratome across a variety of surgical skill levels and does not require advanced levels of training to achieve this consistency.

Keywords: postoperative endophthalmitis, keratome, corneal incision

\section{Introduction}

Postoperative endophthalmitis (POE) is a devastating complication of cataract surgery. Historically, a number of large studies have cited rates between $0.072 \%$ and $0.093 \%$ of POE. ${ }^{1-3}$ Unfortunately, over the past 20 years, there has not been a clear downward trend of POE; and a handful of studies have demonstrated increased rates, up to $0.26 \%{ }^{3}$

Risk factors for the development of POE are multifactorial and include pre- or intra-operative antibiotic prophylaxis, proper surgical technique, and postoperative surveillance. However, there is evidence that clear corneal incision in cataract surgery is itself a risk factor for POE. In Germany a survey of 340,633 cataract surgeries found use of clear corneal incisions as a POE risk factor when compared to corneoscleral incisions. ${ }^{4}$ In Japan a prospective, randomized study of 11,595 small incision cataract surgeries found a relative risk of POE to be 4.6 when temporal corneal incisions were used compared to superior corneoscleral incisions. ${ }^{5}$ An explanation for these increased rates of POE is the possibility of contamination of the wound in the early postoperative period that occurs based on poor wound architecture.

The present diamond keratome design makes creating a perfect incision into the anterior chamber difficult, even in the most skilled hands. Specific concerns with the diamond keratome include: tilt of the keratome as it enters the eye causing asymmetric 


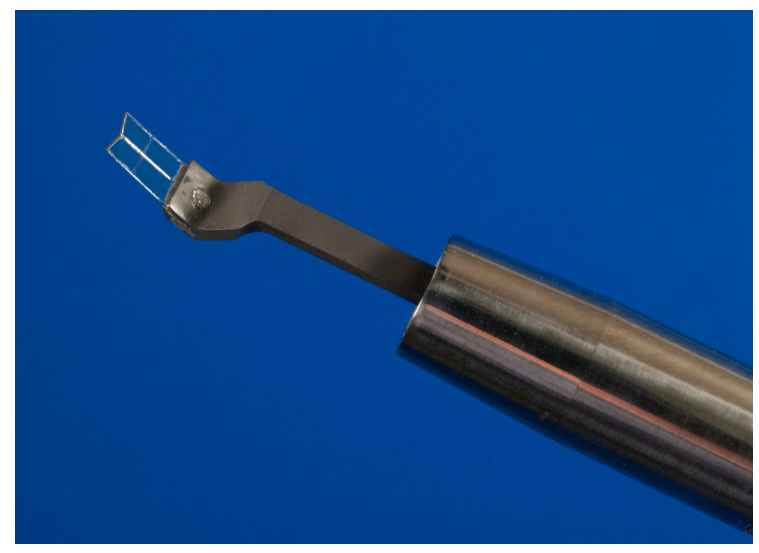

Figure I The newly designed diamond keratome.

wound edges, conjunctival entry that can result in conjunctival ballooning during surgery, extension of the wound secondary to inadvertent patient movement, and tearing of the wound edges during surgical maneuvers in the eye. The net result is that imperfect incisions can and often do result. These imperfect incisions may predispose the corneal incision to leak and potentially result in POE. Wallin et al demonstrated that a leaky wound on postoperative day 1 had the greatest association with POE (increased the risk 44 fold). ${ }^{6}$

Recognizing that tilting is very difficult to completely avoid, the concept of a new blade (Figure 1) is based on the idea that the cutting tip is the surgical focus point, such that the actual right and left edges cannot be controlled and will always be uneven with any blade tilting. Furthermore, as the blade advances, it could always cut to either side if the eye was to move, and it will cut the entrance edges if it is lifted during the final stage of entry into the anterior chamber. The new blade cuts on the outer edges to start and is sharp on the outer edge for only the first 200 microns, after which the edge is smooth and round. The hypothesis is that the incision start will be when both points are placed as desired, so there is no guessing about this; and once the blade is beyond 200 microns into the cornea, it cannot cut to either side and so will track quite precisely. Furthermore, when the desired length is reached, the blade can be lifted with little risk of edge tear-out. This should make the creation of a perfect incision an easier task, especially for those not experienced with using a diamond keratome.

This study is designed to see if this newly designed diamond keratome can improve the structural consistency of the clear corneal incision.

\section{Material and methods}

This study used 15 human donor eyes obtained from the San Diego Eye Bank (San Diego, CA, USA). All eyes collected for this study were specifically donated as research eyes and are provided under our institutional review board approval. The age of the donors ranged from 59 to 88 years. The interval between death and preservation ranged from 3 hours and 47 minutes to 12 hours and 25 minutes. After storing the globes in moist chamber medium, Eye Bank staff shipped them overnight in cold storage to the John A. Moran Eye Center. They were refrigerated until evaluation which took place within 3 days of receipt. Upon removing the cadaver eyes from the moist chamber medium, one of the investigators immediately placed them onto a Mandell Eye Mount (Mastel Precision Surgical Instruments, Rapid City, SD, USA). Suction was initiated using the Mandell Eye Mount, and the intraocular pressure (IOP) was raised to a pressure between $20-35 \mathrm{mmHg}$ as measured by a Tono-Pen AVIA (Reichert Technologies, Depew, NY, USA).

The newly designed diamond keratome blade was made from two single facet diamond blades mounted side-by-side with the leading edge of each mounted on the outside. The outer 200 microns of each was sharpened as part of this process.

Three surgeons of varying levels of experience (JMB second-year resident, RJS - third-year resident, DIB - fifthyear resident) each performed ten incisions with the traditional $2.4 \mathrm{~mm}$ diamond keratome and ten incisions with the newly designed keratome, yielding 30 total incisions with each keratome. Each keratome was marked $2.0 \mathrm{~mm}$ from the tip. The four incisions made in each donor eye were created in the following fashion: the tip was engaged perpendicular to the surface and then angled parallel to the cornea until the keratome was advanced to the $2.0 \mathrm{~mm}$ mark. At this point the keratome was angled to penetrate the globe as close to the $2.0 \mathrm{~mm}$ mark as possible. When the surgeon used the traditional blade, he/she took great care not to lift it, so as not to cut either edge on the incision entrance. No such care was taken with the new blade.

With 20× magnification, angled McPherson tying forceps coated with marking pen dye were used to stain the stromal incision to better delineate its architecture. The surgeon who performed the incision then used Vernier calipers accurate to $0.01 \mathrm{~mm}$ and a dual-headed microscope to measure the actual incision dimensions including the epithelial incision deviation from a straight line (Figure 2). Both the individual performing the initial measuring and another study team member, who served as an observer, verified the measurements. Both surgeons looked through the microscope simultaneously and agreed upon the caliber width before recording the measurement. The observer who verified the initial measurements also noted the presence of any corneal surface edge tears. The surgeon who performed the initial measuring then introduced a 30-degree bent Kelman phacoemulsification needle with 


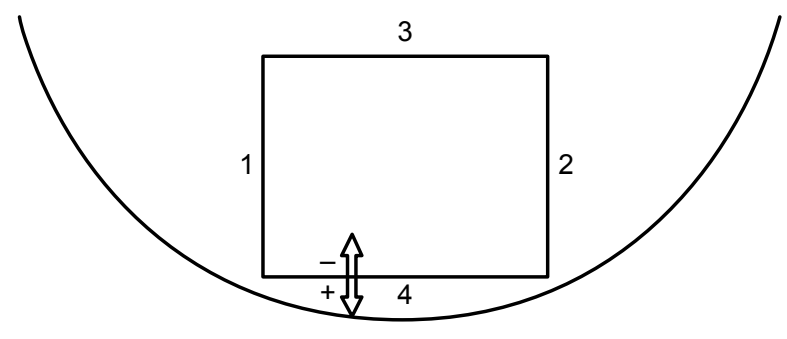

Figure 2 Schematic drawing of the chronology of the clear corneal incision measurements.

Note: I and 2 represent the incision lengths, 3 the internal incision width, and 4 the external incision width and final measurement delineating the posterior wound architecture.

sleeve into the incision, and rotated the tip 60 degrees from the incisional plane in all three potential directions (down, right, and left) to check incision integrity. He/she, as well as the observer, noted and measured any additional corneal surface or internal edge tears. The surgeon who performed the incisions then sealed each one of them with cyanoacrylate glue before making the next incision, in order to be able to restore IOP to the predetermined range.

\section{Statistical analysis}

Study data were collected and managed using Research Electronic Data Capture (REDCap) electronic data capture tools hosted at the University of Utah. REDCap is a secure, web-based application designed to support data capture for research studies. The data were exported to Microsoft Excel 2010 for all statistical analyses. We calculated absolute value of the difference in incision length (right vs left side length; differences indicating the blade was tilted during incision creation) and width (epithelial compared to endothelial incision widths; difference indicating a variable incision size). Means plus standard deviations were determined; and a two sample Student's $t$-test compared the consistency in wound length, width, and epithelial incision variation from a straight line. Significance was set at a $P$-value of $<0.01$ due to multiple comparisons. The difference in incision lengths between the two keratomes was also correlated with experience, and $R^{2}$ values were calculated for both keratomes using a linear regression. This analysis was selected to determine if there was a relationship between experience and consistency of incision architecture.

\section{Results}

Table 1 includes the results of the measurements. The absolute difference between the left and right incision lengths was significantly less with the new blade $(0.182 \pm 0.158 \mathrm{~mm}$ vs $0.088 \pm 0.077 \mathrm{~mm} ; P<0.003$; Table 2). A difference of $0.25 \mathrm{~mm}$ or more was observed eleven times with the traditional keratome with a maximum difference of $0.71 \mathrm{~mm}$, and twice with the new keratome with a maximum difference of $0.28 \mathrm{~mm}$ $(P=0.01)$. Figure 3 displays the mean and standard deviation of the absolute difference between the left and right incision length. With the traditional keratome, this difference correlated with experience but was not statistically significant $\left(R^{2}=0.91 ; P=0.19\right)$, whereas the new keratome did not have this correlation $\left(R^{2}=0.61 ; P=0.43\right)$.

The difference between the epithelial and endothelial incision widths was also more consistent with the new blade compared to the traditional one $(0.080 \pm 0.092$ $\mathrm{mm}$ vs $0.181 \pm 0.144 \mathrm{~mm} ; P<0.002)$. The entry through epithelium with the traditional keratome showed the characteristic "V-type" entry incision line; the positive deviation correlated with the apex of the "V". The negative deviation of the new keratome showed a barely noticeable "U pattern" (traditional keratome $+0.128 \pm 0.242 \mathrm{~mm}$ and new keratome $-0.046 \pm 0.124 \mathrm{~mm} ; P<0.001$, Table 2).

Two incisions with the traditional diamond keratome resulted in epithelial entrance edge tears; both were radial tears with the traditional blade. One occurred with creation of the wound and one occurred after manipulation with the phacoemulsification needle. One edge tear occurred with the newly designed keratome; this was a longitudinal tear, suggesting the tissue was torn rather than cut.

\section{Discussion}

We demonstrated that the newly designed keratome creates a more reliably symmetric wound compared to a traditional diamond keratome, and that the problem of keratome tilting is largely avoidable even for a surgeon who is beginning training. Potentially more important than this difference of left and right side incision lengths $(0.182 \mathrm{vs} 0.088 \mathrm{~mm})$ is the number of cases where the disparity was $0.25 \mathrm{~mm}$ or more (eleven vs two of 30$)$ and the maximum disparity (0.71 vs

Table I Traditional and new keratome mean and standard deviation (SD) of incisions I-4

\begin{tabular}{lllll}
\hline & \multicolumn{2}{l}{ Traditional keratome } & & New keratome \\
\cline { 2 - 4 } & Mean & SD & & Mean \\
\hline Incision I length $(\mathrm{mm})$ & 2.128 & 0.301 & 2.223 & SD \\
Incision 2 length (mm) & 2.178 & 0.330 & 2.221 & 0.317 \\
Incision 3 width (mm) & 2.421 & 0.132 & 2.636 & 0.304 \\
Incision 4 width (mm) & 2.553 & 0.138 & 2.664 & 0.084 \\
\hline
\end{tabular}


Table 2 Traditional and new keratome mean, standard deviation (SD), and $P$-value for the absolute difference in right and left incision lengths, absolute difference in epithelial and endothelial incision width, and epithelial incision central deviation from a straight line

\begin{tabular}{|c|c|c|c|c|c|}
\hline & \multicolumn{2}{|c|}{ Traditional keratome } & \multicolumn{2}{|c|}{ New keratome } & \multirow[t]{2}{*}{$P$-value } \\
\hline & Mean & SD & Mean & SD & \\
\hline Difference in incision length $(\mathrm{mm})$ & 0.182 & 0.158 & 0.088 & 0.077 & $P<0.003$ \\
\hline Difference in incision width (mm) & 0.181 & 0.144 & 0.080 & 0.092 & $P<0.002$ \\
\hline Epithelial incision variation $(\mathrm{mm})$ & 0.128 & 0.242 & -0.046 & 0.124 & $P<0.001$ \\
\hline
\end{tabular}

$0.28 \mathrm{~mm}$ ). A $0.71 \mathrm{~mm}$ difference is likely to be a wound with a great propensity to leak. This study confirms that traditional blade tilting is common and can lead to an edge of the wound that is very short and likely to leak. This has implications for improved wound architecture that also might help decrease rates of POE. If the preoperative sterility and intraoperative sterility remain constant, contamination could be expected to occur in the early postoperative period with wound microleaks. ${ }^{7}$ Many other authors' support this theory as important in understanding the etiology of POE. ${ }^{8,9}$

Tilting can also cut into conjunctiva on one side, and it is the cause of conjunctival ballooning during surgery. While not the subject of this study, the avoidance of blade tilt with the new blade should avoid this problem as well.

Another way to render an incision likely to leak is to tear either edge of the incision during the surgery or with the blade during incision creation. It is felt that one cannot lift a diamond blade but must depress the tip during final entry into the anterior chamber or the epithelial incision edges will be cut. The authors succeeded in avoiding this common complication, which is especially noteworthy for an inexperienced surgeon with only one such cut out of 60 incision edges created. Although it was assumed that no such care was necessary with the new blade, with enough

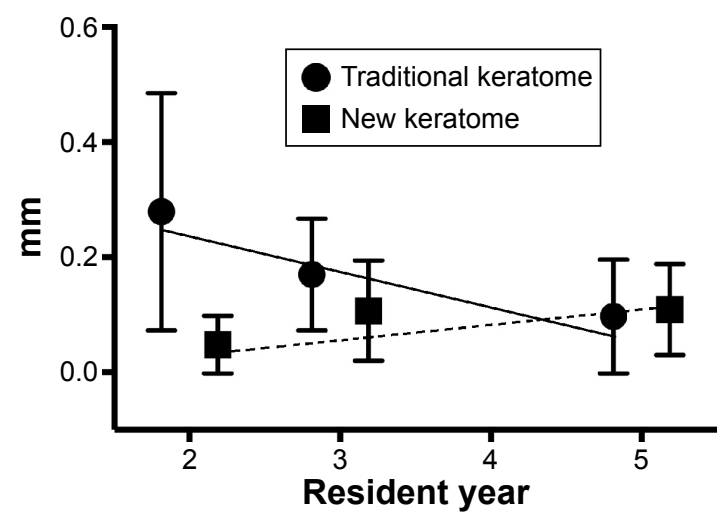

Figure 3 Mean and standard deviation of the absolute difference between the left and right incision length with use of the traditional and new keratomes.

Notes: The horizontal axis shows the number of years of residency training of the study surgeons. Linear regression reveals a non-statistically significant correlation between years of experience and difference in incision lengths when using the traditional keratome (solid line, $R^{2}=0.91$ ), but no such correlation with the new keratome (dashed line, $R^{2}=0.6 \mathrm{I}$ ). force one edge did tear out and was clearly not cut. It is assumed that a little experience should provide the skills to avoid actually tearing the tissue, while the cutting of the edge with a sharp diamond blade is both easy to perform and is often the reason why novices give up on traditional diamond blades early in the learning process. Simulation of surgery did create an additional tear only with the traditional blade. Measuring the tear force needed with a strain gauge, and/or a clinical study, will be necessary to see if the new blade also renders an incision less likely to tear during surgery.

While there was no statistically significant difference in incision edge tears in this study, the movement of a patient's eye during incision creation certainly can result in irregular and even devastating corneal cuts. As a result of the new keratome's design, once the blade advances beyond the first $0.2 \mathrm{~mm}$ cutting edge, this complication should be avoidable.

Another difference in the wounds created by the traditional keratome and the new keratome was the architecture of the epithelial entrance. The new keratome created very few wounds with any anterior or posterior variance. At this time there is no evidence in the literature to support increased leaking related to the irregular epithelial entrance. However, assuming the structural integrity is largely determined by the incision edges, one could hypothesize that the less lip is present, the less likely the wound would leak. Another potential consideration is surgically induced astigmatism (SIA). Studies have demonstrated that smaller incisions induce less astigmatism, ${ }^{10}$ but there are little data regarding the effect that a more symmetric wound may have on SIA. We envision future study of SIA.

Certainly, there are many variables that are different when performing procedures on cadaveric eyes in a laboratory, compared to performing surgery on living patients. While we controlled the pressure in the eyes by using the mounting system and suction, the anterior chambers were not filled with viscoelastic material, as would normally be the case during cataract surgery. Furthermore, for the most part we avoided eye movement. This does not happen in surgery where even with a retaining ring, the eye often moves in the direction of the blade movement, perhaps making blade tilt more difficult to avoid. In addition, while there has been significant research 
examining the effect of wound architecture on leakage, ${ }^{11-15}$ most of these studies have focused on the technique or instrument material used to create the incision and not on actual consistency in incision length or width.

This study was an initial proof of design to determine whether the new keratome is advantageous in creating symmetric and reliable wounds, when compared to the traditional keratome. We included several different experience levels of surgeons in training, and we tried to duplicate the clinical situation to the extent possible. Nonetheless, the most important study limitation is that this is a cadaver eye study and not actual surgery on a living individual. By using human globes and controlling IOP, we feel that we carefully simulated the actual clinical situation and consider our results to be clinically relevant. In the clinical setting, we have used the new blade in 20 consecutive cataract patients without any difficulty and all incisions were seen to be very consistent. Furthermore, there were no cases of conjunctival ballooning even though the entry was right at the conjunctival insertion.

By cutting with only the first $0.2 \mathrm{~mm}$ on the outer edge and centrally, we created more consistent incisions with the new keratome design when compared to a traditional blade design. We also demonstrated that a high level of experience is not needed to perform this procedure. Furthermore, we documented decreased blade tilt as well as a more linear epithelial entrance. Future studies include assessment of the possibility of incision leakage differences in hypotonous eyes, anterior chamber optical coherence tomography to examine microscopic wound architecture, and determination of differences that may occur with SIA. We will also evaluate incision edge tear strength by using a strain gauge to determine whether the new blade creates an incision that will be more resistant to tearing during surgery. Clinical studies will be required in order to determine whether incisions are less likely to leak with the new keratome design, and whether this design impacts POE incidence.

\section{Acknowledgments}

The staff of the San Diego Eye Bank prepared and shipped the human donor eyes that were used for this study. Susan Schulman, MAT, School of Medicine, University of Utah, provided writing and editing assistance.

\section{Financial and material support}

This study was supported in part by a Center for Clinical and Translational Sciences grant to the University of Utah
(8UL1TR000105 [formerly UL1RR025764] NCATS/NIH). This study also was supported in part by an unrestricted grant from Research to Prevent Blindness, Inc., New York, New York, USA, to the Department of Ophthalmology and Visual Sciences, University of Utah, Salt Lake City, Utah.

\section{Disclosure}

Randall $\mathrm{J}$ Olson has a financial interest in the new blade design. All other authors have no financial or proprietary interests.

\section{References}

1. Aaberg TM Jr, Flynn HW Jr, Schiffman J, Newton J. Nosocomial acute-onset postoperative endophthalmitis survey. A 10-year review of incidence and outcomes. Ophthalmology. 1998;105(6):1004-1010.

2. Kattan HM, Flynn HW Jr, Pflugfelder SC, Robertson C, Forster RK. Nosocomial endophthalmitis survey. Current incidence of infection after intraocular surgery. Ophthalmology. 1991;98(2):227-238.

3. Jensen MK, Fiscella RG. Comparison of endophthalmitis rates over four years associated with topical ofloxacin vs ciprofloxacin. Invest Ophthalmol Vis Sci. 2002;43(12):4429.

4. Schmitz S, Dick HB, Krummenauer F, Pfeiffer N. Endophthalmitis in cataract surgery: results of a German survey. Ophthalmology. 1999; 106(10):1869-1877.

5. Nagaki Y, Hayasaka S, Kadoi C, et al. Bacterial endophthalmitis after small-incision cataract surgery. effect of incision placement and intraocular lens type. J Cataract Refract Surg. 2003;29(1):20-26.

6. Wallin T, Parker J, Jin Y, Kefalopoulos G, Olson RJ. Cohort study of 27 cases of endophthalmitis at a single institution. J Cataract Refract Surg. 2005;31(4):735-741.

7. Olson RJ. Reducing the risk of postoperative endophthalmitis. Surv Ophthalmol. 2004;49 Suppl 2:S55-S61.

8. Vasavada AR, Praveen MR, Pandita D, et al. Effect of stromal hydration of clear corneal incisions: quantifying ingress of trypan blue into the anterior chamber after phacoemulsification. J Cataract Refract Surg. 2007;33(4):623-627.

9. Herretes S, Stark WJ, Pirouzmanesh A, Reyes JM, McDonnell PJ, Behrens A. Inflow of ocular surface fluid into the anterior chamber after phacoemulsification through sutureless corneal cataract wounds. Am J Ophthalmol. 2005;140(4):737-740.

10. Moon SC, Mohamed T, Fine IH. Comparison of surgically induced astigmatisms after clear corneal incisions of different sizes. Korean J Ophthalmol. 2007;21(1):1-5.

11. May WN, Castro-Combs J, Quinto GG, Kashiwabuchi R, Gower EW, Behrens A. Standardized Seidel test to evaluate different sutureless cataract incision configurations. J Cataract Refract Surg. 2010;36(6): 1011-1017.

12. Masket S, Belani S. Proper wound construction to prevent short-term ocular hypotony after clear corneal incision cataract surgery. J Cataract Refract Surg. 2007;33(3):383-386.

13. Hayashi K, Tsuru T, Yoshida M, Hirata A. Intraocular pressure and wound status in eyes immediately after scleral tunnel incision and clear corneal incision cataract surgery. Am J Ophthalmol. 2014;158(2): 232-241.

14. Jacobi FK, Dick HB, Bohle RM. Histological and ultrastructural study of corneal tunnel incisions using diamond and steel keratomes. J Cataract Refract Surg. 1998;24(4):498-502.

15. Etter J, Berdahl J, Jun B, Caldwell M, Kim T. Corneal wound integrity and architecture after phacoemulsification: comparative analysis of corneal wounds created by silicon and steel blades. J Cataract Refract Surg. 2009;35(7):1313-1314. 


\section{Publish your work in this journal}

Clinical Ophthalmology is an international, peer-reviewed journal covering all subspecialties within ophthalmology. Key topics include: Optometry; Visual science; Pharmacology and drug therapy in eye diseases; Basic Sciences; Primary and Secondary eye care; Patient Safety and Quality of Care Improvements. This journal is indexed on

PubMed Central and CAS, and is the official journal of The Society of Clinical Ophthalmology (SCO). The manuscript management system is completely online and includes a very quick and fair peer-review system, which is all easy to use. Visit http://www.dovepress.com/ testimonials.php to read real quotes from published authors. 